\title{
Publisher Correction: Reassessing apoptosis in plants
}

Martin Dickman, Brett Williams, Yurong Li, Paul de Figueiredo and Thomas Wolpert

Correction to: Nature Plants https://doi.org/10.1038/s41477-017-0020-x (2017); published online 25 September 2017.

In the version of this Perspective originally published, the name of co-author Paul de Figueiredo was incorrect. This has now been corrected.

Published online: 16 October 2017

https://doi.org/10.1038/s41477-017-0045-1 